\title{
Traumatic Thoracic Aorta Injuries: Outcomes up to 15 years Post Thoracic Endovascular Aortic Repair
}

\author{
Varun J Sharma MBBS', Martin J Jarmin MD², John A Crozier FRACS', \\ Saksham Gupta MBBS ${ }^{1}$ and Jim N Iliopoulos PhD FRACS ${ }^{1,3}$
}

\author{
'Department of Vascular Surgery, Liverpool Hospital, Liverpool, New South Wales, Australia \\ 2Department of Trauma Surgery, Liverpool Hospital, Liverpool, New South Wales, Australia \\ ${ }^{3}$ Department of Surgery, University of New South Wales, Liverpool, New South Wales, Australia
}

\begin{abstract}
Background: Aortic injuries are a leading cause of death following trauma, with $75 \%$ pre- and 50\% in-hospital mortality. Endovascular repair is technically easier with fewer complications but long-term results are unproven. Methods: A retrospective analysis of patients with endovascular repair of thoracic aortic injuries from 2001 to 2018 at Liverpool Hospital, Sydney, Australia was undertaken. Primary endpoint was death and secondary endpoints were re-interventions, hand ischemia, access vessel repair and ischemic complications.

Results: 24 patients (10 female) were reviewed, the most common mechanism of injury being motor-vehicle related $(75 \%)$ in Zone $3(71 \%)$. Deployment was proximal $(n=11)$, on $(n=2)$ or distal $(n=11)$ to the left subclavian artery (LSCA). Average follow-up was $5.4 \pm 5.1$ years (range 0.1-15.2 years), with two deaths. At $<90$ days, complications were hand ischemia ( $n=4, n=1$ needing intervention), access vessel endarterectomy $(n=1)$ and conversion to open bypass $(n=1)$. At $>90$ days, complications were hand ischemia $(n=1)$, graft migration $(n=2)$ and minor graft thrombosis $(n=1)$. Coverage of LSCA was not a predictor of re-intervention $(P=0.43)$ or supra-aortic bypass $(P=$ 0.13 ). Survival free from reintervention in the non-covered LSCA group was $100 \%$ at the 30 -day and 6-month timepoints, and $80 \%$ at the 1 -year and 5 -year timepoints. Survival free from reintervention in the covered LSCA group was $84 \%, 75.6 \%, 67.2 \%$ and $67.2 \%$ at the 30-day, 6-month, 1-year and 5-year timepoints, respectively.

Conclusions: Endovascular repair for aortic injuries has low levels of morbidity. The LSCA can be covered without arm ischemia and is not predictive of re-intervention or a supra-aortic bypass. At up to 15 years follow-up, graft complications remain low.
\end{abstract}

Keywords: Trauma; Aorta; Endovascular; Stent

Received: 19 November 2019; Accepted: 17 December 2019

\section{INTRODUCTION}

Aortic injuries are one of the leading causes of death following trauma, with a $75 \%$ pre-hospital and $50 \%$ in-hospital mortality [1,2]. The aortic isthmus is the

\section{Corresponding author:}

Department of Vascular Surgery, Liverpool Hospital, Liverpool, Sydney, Australia.

Email: varun_sharma@hsph.harvard.edu

Presentation: This data was presented at the Royal Australasian College of Surgeons Annual Scientific Congress, Bangkok,

Thailand, $10^{\text {th }}$ May 2019.

(C) 2020 CC BY 4.0 - in cooperation with Depts. of Cardiothoracic/ Vascular Surgery, General Surgery and Anesthesia, Örebro University Hospital and Örebro University, Sweden location for $90 \%$ of injuries, attributed to the osseous pinch theory, caused by a sudden deceleration and traction of the immobile isthmus at the junction of the mobile ascending arch and the fixed descending aorta combined with the shear stress between anterior and posterior bony structures [3].

The most common mechanism is motor-vehicle injuries or rarely blunt trauma from falls. The high degree of force needed generally results in a large number of concomitant injuries, in particular cardiac and pulmonary contusions, thoracic cage fractures and significant hemodynamic instability, all of which confer a high operative risk when open surgery is performed. Such intervention has historically required thoracotomy with single lung ventilation, cardiopulmonary bypass, systemic anticoagulation and aortic cross clamping, and is fraught by paraplegia from spinal cord ischemia, delayed rupture and mortality. 
The advent of endovascular techniques for thoracic aortic repair (TEVAR) in 1991 and their first use in trauma in 1997 have been met with optimism, with improved perioperative morbidity and mortality [2,4-8]. However, there remains uncertainty regarding the timing of TEVAR in stable patients, management of minimal aortic injuries (periadventitial defects or hematomas), the role for prophylactic spinal drainage, choice of anesthesia and optimal follow-up imaging modality, with the level of evidence to substantiate the last series of recommendations from the Society of Vascular Surgery (SVS) remaining poor (Level IIC) $[1,9,10]$.

In particular, as most interventions are undertaken on young patients (median age 39), there are concerns regarding the long-term stent conformation to the inferior part of the aortic arch, sequalae of left subclavian artery (LSCA) coverage, effects of age-related morphological changes, cumulative radiation exposure in follow-up, material failures, fractures and fabric fatigue [11-15].

\section{METHODS}

Patients with TEVARs undertaken for trauma were identified using the Australian Vascular Audit, the Liverpool Hospital electronic and paper medical records and the Liverpool Hospital Trauma Database.

Retrospective analysis was undertaken to determine demographic, operative and follow-up data. The primary endpoint measured was death. Secondary endpoints were conversion to open and take back operations, hand ischemia, acute surgical complications to access vessels, cardiopulmonary complications, stroke, spinal cord ischemia and end organ dysfunction. Stents were analyzed for endoleak, graft migration, collapse and thrombosis. Statistical analyses were performed with Microsoft Excel (2016) and Stata (v15.1).

\section{Ethical Approval and Informed Consent}

As a retrospective study, ethics was approved by the Research and Ethics Department of Liverpool Hospital, Liverpool, Australia (LNR/18/LPOOL/530) in lieu of individual informed consent.

\section{RESULTS}

Since 2001, there were 24 patients who underwent thoracic stenting for traumatic aortic injuries. The average age at operation was 47.9 years (Table 1), and the most common mechanism of injury was motor-vehicle related $(75 \%)$ (Figure 1). The presence of previous co-morbidities was low and the average Injury Severity Score (ISS, 2015) [4, 16] on arrival was 35 (Table 1).

The median time to intervention was 1.0 days (range 0-100 days). Common concurrent injuries were thoracic contusions, pneumo-hemothoraces and rib, pelvic, scapular, clavicular and vertebral fractures (Figure 1). Median
Table 1 Patient demographics.

\begin{tabular}{ll}
\hline Patients $[n(\%)]$ & $14(58 \%)$ \\
$\quad$ Male & $10(42 \%)$ \\
Female & $47.9 \pm 16.1$ years \\
Age (range) & $(26-84$ years) \\
& \\
Co-morbidities [n (\%)] & $7(29 \%)$ \\
Hypertension & $5(21 \%)$ \\
Type 2 diabetes & $5(21 \%)$ \\
Ischemic heart disease & $6(25 \%)$ \\
Smoking & $3(12 \%)$ \\
Intravenous drug use & $35.3 \pm 13.6(16-59)$ \\
Injury Severity Score (ISS) & $11.2 \pm 10.8$ days \\
Average length of intensive care unit stay & $(5-468$ days) \\
& $59.7 \pm 93.9$ days \\
Average length of hospital stay & $(2-40$ days) \\
& \\
Mechanism of injury [n (\%)] & $21(87.5 \%)$ \\
Motor-vehicle/cycle injury & $3(12.5 \%)$ \\
Fall &
\end{tabular}

intensive care unit and hospital stay was 4.5 (interquartile range (IQR) 3.0-19.0) and 27.5 (IQR 14.8-82.3) days, respectively.

Using the SVS guidelines $[1,9,10]$, all patients with a Grade III (pseudoaneurysm, $n=13$ ) and Grade IV (disruption with rupture, $n=11$ ) injuries underwent TEVAR, which were located in Zone $2(n=3)$, Zone $3(n=17)$ and Zone $4(n=4)$ (Figure 2).

All grafts were Zenith Thoracic grafts (Cook Medical), with graft diameter ranging from 24-38 mm. Access was either percutaneously with a closure device $(n=11)$ or via femoral cutdown $(n=13)$. Proximal landing zone was proximal to the LSCA in 11 patients, partially covering the LSCA in 2 patients and distal to LSCA in 11 patients. In patients with LSCA covering, two patients had LSCA coiling of which one also had a prophylactic left common carotid artery (LCCA) to LSCA bypass. The classification of endoleak was defined similarly to contemporary definitions for aneurysmal pathology; Type I endoleak was defined as a lack of a proximal or distal stent graft seal, including retrograde flow from the LSCA; Type II endoleak was classified as any other retrograde filling from arteries of the descending thoracic aorta. Significant Type I endoleak was noted intraoperatively in three patients, and Type II in one patient. The distal landing zone in all patients was the distal thoracic aorta, just above the coeliac axis.

In the immediate postoperative period (up to 90 days, Table 2), there were no deaths. Indications for reintervention were hand ischemia ( $n=3, n=1$ requiring urgent intervention within 24 hours), access vessel occlusion needing endarterectomy $(n=1)$ and pseudoaneurysm formation with Type Ia endoleak ( $n=1$, requiring conversion to open bypass). There was high concurrence of cardiac $(n=2)$, thoracic $(n=12)$ and acute renal failure $(n=7)$ complications, but these were not iatrogenic. 
(a)
- Motor Vehicle Accident
- Motor Cycle Accident
- Fall from Height $>3 \mathrm{~m}$
- Quad Bike Accident

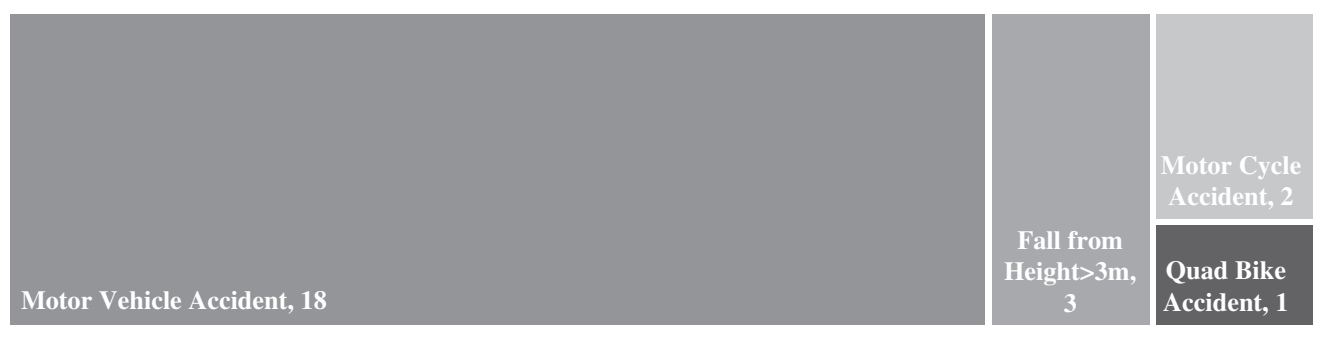

(b)

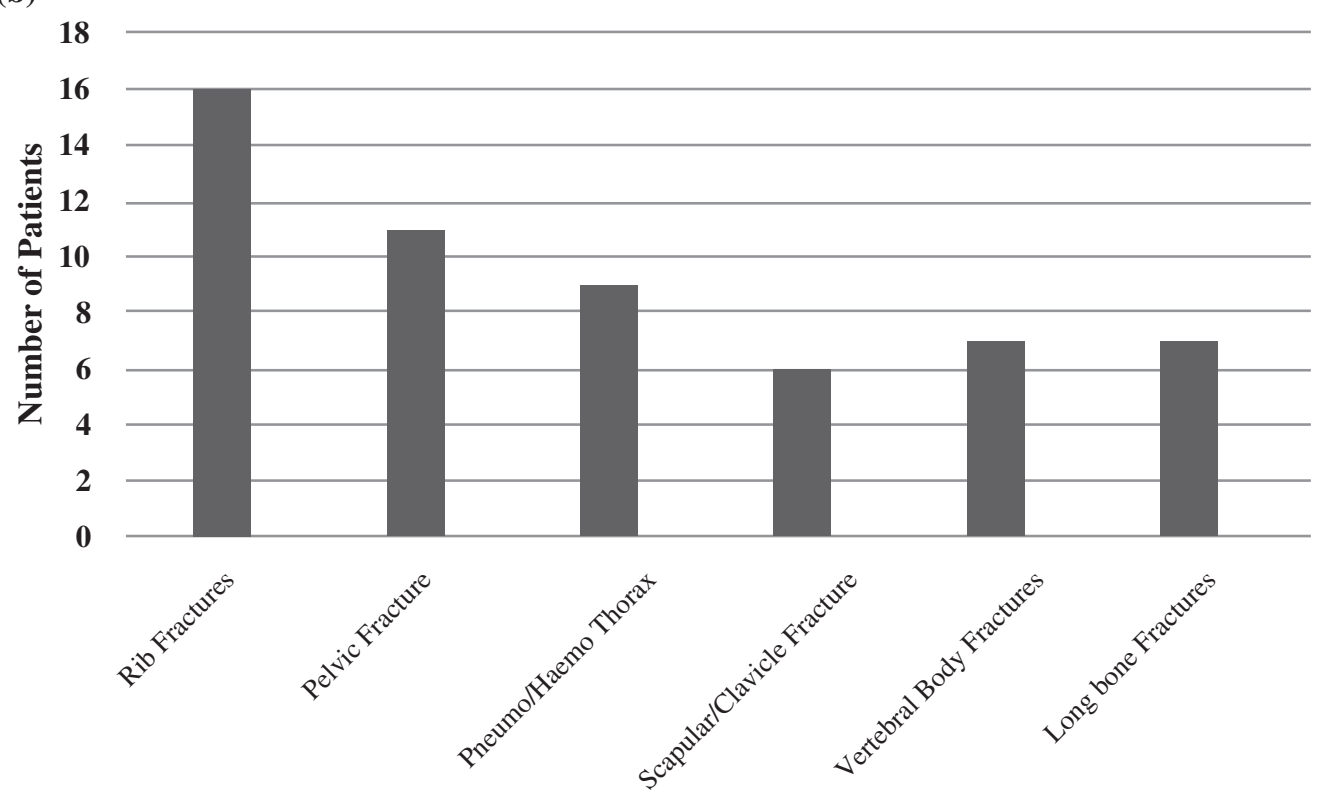

Figure 1 Mechanisms of injury and common concurrent injuries. (a) Mechanism of injury. Motor-vehicle injury formed the majority of cases $(n=18)$ followed by fall from height $(n=3)$, motorcycle injuries $(n=2)$ and four-wheel quad bike injuries $(n=1)$. (b) Common concurrent injuries in patients who underwent endovascular repair for traumatic aortic injuries.

There were no episodes of spinal cord ischemia, stroke or paraplegia secondary to endovascular intervention.

Post discharge (greater than 90 days, Table 2), average follow-up time was 5.4 years with maximal follow-up 15.2 years (Figure 3 ). There were two deaths; one from graft sepsis and one from intracerebral hemorrhage post fall for an unrelated admission (the patient was not anticoagulated). Of the patients with persistent hand ischemia (claudication) post 90 days $(n=3)$, one needed LCCA to LSCA bypass at 9 months, and the remaining two patients' symptoms resolved with physiotherapy. There was no graft thrombosis, but one patient required iliac intervention ipsilateral to the site of femoral access, and another one showed evidence of non-flow limiting stenosis at 10 years follow-up. Graft migration was noted in two patients, and there was no evidence of aortic dilation.

Coverage of the LSCA did confer any statistically significant risk on re-intervention or the need for a LCCA to
LSCA bypass (Table 3 and Figure 3). Although a greater number of patients with stent placement proximal to the LSCA required LCCA to LSCA bypass ( $n=3 / 11$ vs. $n=0 / 11)$, this difference was not statistically significant $(P=0.13$, Figure 3$)$. The survival free from reintervention in the non-covered LSCA group was $100 \%$ at the 30 -day and 6-month timepoints, and $80 \%$ at the 1 -year and 5-year timepoints. The survival free from reintervention in the Covered LSCA group at was $84 \%, 75.6 \%$, $67.2 \%$ and $67.2 \%$ at the 30-day, 6-month, 1-year and 5 -year timepoints, respectively. The survival free from LCCA-LSCA bypass in the non-covered LSCA group was $100 \%$ at all timepoints; in the covered LSCA group it was $92.0 \%, 83.7 \%, 83.7 \%$ and $57.4 \%$ at the 30 -day, 6-month, 1-year and 5-year timepoints, respectively. Concurrent injuries and the zone of injury did not confer any statistically significant risk of re-intervention or bypass. 
(a)

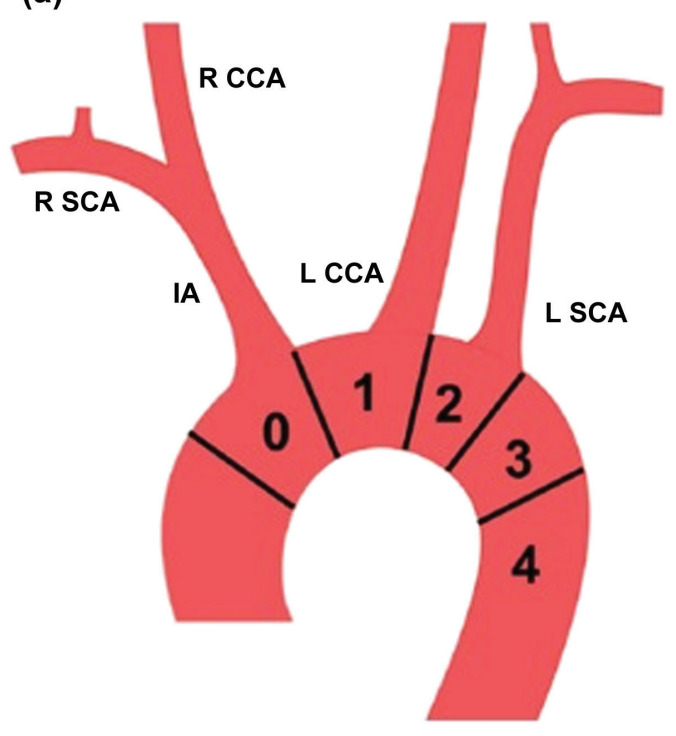

(b)

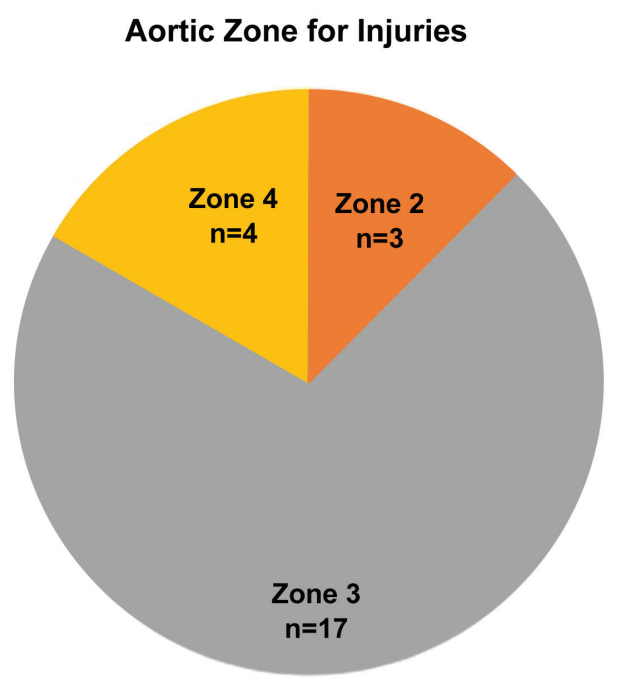

Figure 2 Zones of aortic injuries. (a) Schematic to classify zones of aortic Injury. RSCA, right subclavian artery; RCCA, right common carotid artery; LCCA, left common carotid artery; LSCA, left subclavian artery; IA, innominate artery. (b) Representation of zones of aortic injuries in this case series.

Table 2 List of complications post operatively, stratified into the post-operative period ( $<90$ days) and post-discharge period ( $>90$ days).

\begin{tabular}{lllll}
\hline \multirow{2}{*}{ Complication } & \multicolumn{2}{c}{ Number of Patients } & \multicolumn{2}{c}{ Number of Patients Needing Intervention } \\
\cline { 2 - 5 } & $<90$ days & $>90$ days & $<90$ days & $>90$ days \\
\hline Type I endoleak & 3 & 0 & 1 & 2 \\
Type II endoleak & 1 & 0 & 0 & 0 \\
Pseudoaneurysm formation & 1 & 0 & 1 & 0 \\
Access vessel damage & 2 & 0 & 1 & 0 \\
Graft infection & 0 & 1 & 0 & 1 \\
Hand ischemia & 4 & 0 & 1 & 1 \\
Graft migration & 0 & 2 & 0 & 0 \\
\hline
\end{tabular}

In the first 2 years of this therapy being undertaken at our institute, there were three patients with untreated Type I endoleak which were not initially treated, all of whom needed reintervention (Tables 2 and 3). Strategies employed thereafter to negate intra-operative endoleak included coiling of the LSCA $(n=2)$ or covering the endoleak with a second stent $(n=1)$, both of which were undertaken with no complications or hand ischemia. We did not observe any strokes, vertebral artery compromise or posterior circulation symptoms after covering the LSCA with a stent graft.

\section{CONCLUSIONS}

Thoracic aortic injuries most commonly occur at the aortic isthmus $(n=17,70.8 \%)$ in motor-vehicle injuries $(n=18,75 \%)$. The rates of concurrent injuries are high (Figure 1), with high trauma scores (average ISS $=35.3 \pm$ 13.6) and lengthy inpatient admissions (average inpatient stay $59.7 \pm 93.9$ days). Anecdotally, we found endovascular repair of traumatic thoracic aortic injuries is technically easier, less invasive and negates the need for invasive and lengthy operations such as cardiopulmonary bypass, systemic anticoagulation and aortic cross clamping.

As a primary endpoint, we report a much lower rate of death at $8 \%(n=2)$ versus the literature rate of $28 \%$ for open repair. There were other significant risk factors for both deaths in our series; the first death was due to graft infection in a patient with ongoing intravenous drug use, and the second was for intracerebral hemorrhage post fall in an unrelated admission (the patient 
(a)

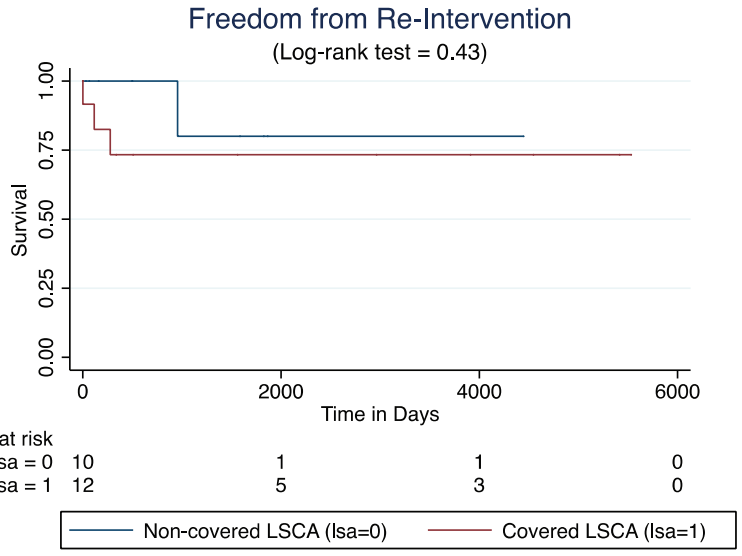

(b)

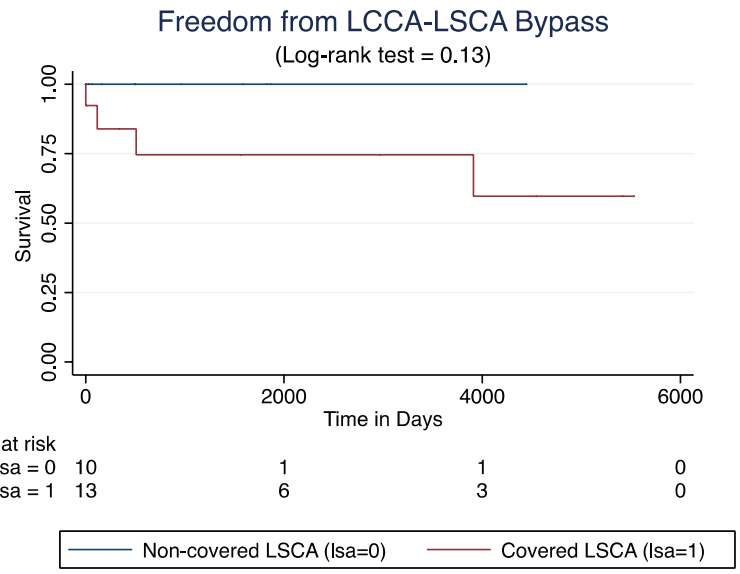

Figure 3 Time to event analysis following intervention. (a) Kaplan-Meier curve demonstrating freedom from RE-intervention with follow-up to 15.2 years. (b) Kaplan-Meier curve demonstrating freedom from left common carotid artery to left subclavian artery (LCCA-LSCA) bypass with follow-up to 15.2 years. The difference was not statistically significant ( $P$-value of log-rank test $=0.43$ and 0.13 , respectively) between those with and without LSCA coverage.

was not anti-coagulated). The secondary endpoint of re-intervention is low in both the post-operative period (<90 days) $(n=4,16.7 \%)$ and long-term follow-up ( $<90$ days) $(n=4,16.7 \%)$. Only two of these patients required a conversion to open repair. The first was a patient with ongoing intravenous drug use who developed graft sepsis requiring graft explant. The second patient had a pseudoaneurysm formation at the site of Type I endoleak, needing an open explant and repair with interposition graft. Rates of other endpoints were also low; in open repair, the literature reports paraplegia from spinal cord ischemia in $16 \%$ and delayed rupture in $5 \%$ of patients, whilst we had no such complications at our center [5-8].

The safety of covering the LSCA in the thoracic aorta remains controversial in the literature. In our series, all patients who developed hand ischemia $(n=4)$ had either complete or partial coverage of the LSCA, but this difference was not statistically significant and is in keeping
Table 3 Predictors of re-intervention and LCCA-LSCA bypass.

\begin{tabular}{lll}
\hline Factor & $\begin{array}{l}\text { Relative Risk } \\
(95 \% \text { Confidence Interval) }\end{array}$ & P-Value \\
\hline Re-intervention & & \\
Any coverage of LSCA & $2.54(0.31,21.06)$ & 0.3882 \\
Complete coverage of SCA & $3.27(0.40,27.00)$ & 0.2708 \\
ICU LOS $>5$ days & $1.18(0.20,7.06)$ & 0.8547 \\
Any endoleak & $15.00(2.05,110.00)$ & 0.0077 \\
Type 1 endoleak & $21.00(3.10,142.21)$ & 0.0018 \\
Type 2 endoleak & $1.71(0.13,22.82)$ & 0.6832 \\
& & \\
LCCA-LSCA bypass & & \\
Any coverage of LSCA & $3.38(0.44,26.00)$ & 0.2412 \\
Complete coverage of SCA & $4.73(0.62,36.32)$ & 0.1354 \\
Any endoleak & $3.33(0.80,13.95)$ & 0.0992 \\
Type I endoleak & $4.67(1.25,17.43)$ & 0.0220 \\
Type II endoleak & $1.09(0.09,13.43)$ & 0.9458 \\
\hline
\end{tabular}

ICU, intensive care unit; LCCA, left common carotid artery; LSCA, left subclavian artery; LOS, length of stay; SCA, subclavian artery.

with findings from the literature to date $(P=0.13)$. Two patients $(15.4 \%)$ needed reintervention with a left common carotid to subclavian bypass (at day 1 , and 9 months post operatively). The majority of patients $(69.2 \%, n=9 / 13)$ who had some or complete coverage of the LSCA did not develop symptoms and did not need re-intervention or a bypass. We therefore believe that covering the subclavian artery remains safe, albeit with risk of hand ischemia which will likely remain amenable to non-surgical interventions such as physiotherapy.

The long-term durability of the graft, irrespective of zone, remains good at an average of 5.4 years and up to 15 years follow-up. Patients with no arch involvement (Zone 4) remained complication free, and only minor evidence of graft migration $(n=2)$ and non-flow limiting graft thrombosis $(n=1)$ was seen in patients with some arch involvement (Zones 2 and 3), all of which did not necessitate reintervention. Many of these complications have been addressed with newer generation of stents which have demonstrated improved hemodynamics and conformation to the aortic arch when deployed for aneurysmal disease $[8,14,15]$. The early complications with Type I endoleak highlight the learning curve in employing this treatment strategy. At our center, we have used coiling of the LSCA or covering the endoleak with a second stent as methods to prevent endoleak and did not observe any such complications after the first 2 years of our experience.

There are several limitations to this study. First, at an average age of 47 years, but with a standard deviation of 16 years, and a range from $26-84$ years, it is difficult to assess the initial goal of assessing age-related differences between open repair and true TEVAR. Second, given that the average age of intervention is generally under 50 years in patients with otherwise unremarkable 
co-morbidities in both our study and the literature (Table 1), the long-term durability of this graft at greater than 20 years still necessitates further investigation. Finally, there also remains no consensus on how longterm follow-up should be undertaken. The majority of our patients underwent yearly or second yearly computed tomography scans, but with an average age of 47 years and low rates of graft complications $(n=3)$, this may not only be unnecessary, but also place patients at high risk of radiation and contrast exposure. Other methods of follow-up include ultrasound or echocardiogram, but the anatomical position of stents renders appropriate ultrasonographic imaging difficult, and most interpretations need to be obtained indirectly through vertebral artery or upper limb flow analyses. Routine X-rays, whilst having less exposure, do not give any functional flow information. It is hoped that, with further studies with long-term follow-up data, such guidelines can be constructed.

\section{Ethics Statement}

(1) All the authors mentioned in the manuscript have agreed to authorship, read and approved the manuscript, and given consent for submission and subsequent publication of the manuscript.

(2) The authors declare that they have read and abided by the JEVTM statement of ethical standards including rules of informed consent and ethical committee approval as stated in the article.

\section{Conflicts of Interest}

The authors declare that they have no conflicts of interest.

\section{Funding}

The authors received no financial support for the research, authorship, and/or publication of this article.

\section{Author Contributions}

VJS carried out the writing and data collection, MJJ the data collection and writing, SG the data collection, JAC the data collection and design, and JNI the data collection, design, and writing.

\section{REFERENCES}

[1] Jamieson WR, Janusz MT, Gudas VM, Burr LH, Fradet GJ, Henderson C. Traumatic rupture of the thoracic aorta: third decade of experience. Am J Surg. 2002; 183(5):571-5.
[2] Cowley RA, Turney SZ, Hankins JR, Rodriguez A, Attar S, Shankar BS. Rupture of thoracic aorta caused by blunt trauma. A fifteen-year experience. J Thorac Cardiovasc Surg. 1990;100(5):652-60; discussion 60-1.

[3] Crass JR, Cohen AM, Motta AO, Tomashefski JF Jr, Wiesen EJ.A proposed new mechanism of traumatic aortic rupture: the osseous pinch. Radiology. 1990;176(3):645-9.

[4] Akhmerov A, DuBose J, Azizzadeh A. Blunt thoracic aortic injury: current therapies, outcomes, and challenges. Ann Vasc Dis. 2019;12(1):1-5.

[5] Jakimowicz T, Rowinski O, Galazka Z, Solonynko B, Szmidt J. Endovascular repair of traumatic thoracic aortic rupture: A single centre experience. Kardiol Pol. 2013; 71(12):1273-8.

[6] Azizzadeh A, Charlton-Ouw KM, Chen Z et al. An outcome analysis of endovascular versus open repair of blunt traumatic aortic injuries. J Vasc Surg. 2013;57(1): 108-14; discussion 115.

[7] Azizzadeh A, Keyhani K, Miller CC 3rd, Coogan SM, Safi HJ, Estrera AL. Blunt traumatic aortic injury: Initial experience with endovascular repair. J Vasc Surg. 2009; 49(6):1403-8.

[8] Azizzadeh A, Ray HM, Dubose JJ et al. Outcomes of endovascular repair for patients with blunt traumatic aortic injury. J Trauma Acute Care Surg. 2014;76(2): 510-16.

[9] Jonker FH, Giacovelli JK, Muhs BE, Sosa JA, Indes JE. Trends and outcomes of endovascular and open treatment for traumatic thoracic aortic injury. J Vasc Surg. 2010;51(3):565-71.

[10] Erben Y, Trejo G, Brownstein AJ et al. Endovascular thoracic aortic transection repair has equivalent survival to open repair after blunt thoracic aortic injury. Int Angiol. 2018;37(2):155-9.

[11] Pang D, Hildebrand D, Bachoo P. Thoracic endovascular repair (TEVAR) versus open surgery for blunt traumatic thoracic aortic injury. Cochrane Database Syst Rev. 2015;9(9):CD006642.

[12] Canaud L, Marty-Ane C, Ziza V, Branchereau P, Alric P. Minimum 10-year follow-up of endovascular repair for acute traumatic transection of the thoracic aorta. J Thorac Cardivasc Surg. 2015;149(3):825-9.

[13] Khoynezhad A, Donayre CE, Azizzadeh A, White R. One-year results of thoracic endovascular aortic repair for blunt thoracic aortic injury (rescue trial). J Thorac Cardiovasc Surg. 2015;149(1):155-61.e4.

[14] Li S, Cai W, Li X, Qiu J, Li Q, Shu C. Thoracic endovascular aortic repair for traumatic type b aortic dissection: a 5-year experience from a single center. Int Angiol. 2017;36(4):316-21.

[15] Canaud L, Cathala P, Joyeux F, Branchereau P, MartyAne C, Alric P. Improvement in conformability of the latest generation of thoracic stent grafts. J Vasc Surg. 2013;57(4):1084-9.

[16] Association for the Advancement of Automotive Medicine (AftAoA): Abbreviated injury scale (AIS) 2015 - Overview. https://www.aaam.org/abbreviated-injury-scale-ais/. Accessed 12 December 2018. 